\title{
Van Allen Probe observations of drift-bounce resonances with Pc 4 pulsations and wave-particle interactions in the pre-midnight inner magnetosphere
}

\author{
G. I. Korotova ${ }^{1,2}$, D. G. Sibeck ${ }^{3}$, K. Tahakashi ${ }^{4}$, L. Dai ${ }^{5}$, H. E. Spence ${ }^{6}$, C. A. Kletzing ${ }^{7}$, J. R. Wygant ${ }^{8}$, \\ J. W. Manweiler ${ }^{9}$, P. S. Moya ${ }^{10}$, K.-J. Hwang ${ }^{3}$, and R. J. Redmon ${ }^{11}$ \\ ${ }^{1}$ IZMIRAN, Russian Academy of Sciences, Moscow, Troitsk, Russia \\ ${ }^{2}$ IPST, University of Maryland, College Park, MD, USA \\ ${ }^{3}$ Code 674, NASA/GSFC, Greenbelt, MD, USA \\ ${ }^{4}$ JHU/APL, Laurel, MD, USA \\ ${ }^{5}$ University of Minnesota, Minneapolis, MN, USA \\ ${ }^{6}$ EOS, University of New Hampshire, Durham, NH, USA \\ ${ }^{7}$ Iowa University, Iowa City, IA, USA \\ ${ }^{8}$ University of Minnesota, Minneapolis, MN, USA \\ ${ }^{9}$ Fundamental Technologies, LLC, Lawrence, KS, USA \\ ${ }^{10}$ Code 673, NASA/GSFC, Greenbelt, MD, USA \\ ${ }^{11}$ NGDC/NOAA, Boulder, CO, USA \\ Correspondence to: G. I. Korotova (gkorotov@umd.edu)
}

Received: 21 January 2015 - Revised: 24 April 2015 - Accepted: 24 June 2015 - Published: 5 August 2015

\begin{abstract}
We present Van Allen Probe B observations of azimuthally limited, antisymmetric, poloidal Pc 4 electric and magnetic field pulsations in the pre-midnight sector of the magnetosphere from 05:40 to 06:00 UT on 1 May 2013. Oscillation periods were similar for the magnetic and electric fields and proton fluxes. The flux of energetic protons exhibited an energy-dependent response to the pulsations. Energetic proton variations were anticorrelated at medium and low energies. Although we attribute the pulsations to a drift-bounce resonance, we demonstrate that the energydependent response of the ion fluxes results from pulsationassociated velocities sweeping energy-dependent radial ion flux gradients back and forth past the spacecraft.
\end{abstract}

Keywords. Solar physics astrophysics and astronomy (wave-particle interactions)

\section{Introduction}

Toroidal and poloidal Pc 4 and Pc 5 pulsations are common in both the dayside and nightside magnetosphere (e.g.,
Arthur and McPherron, 1981; Hughes et al., 1978; Anderson et al., 1990). Toroidal pulsations have been attributed to a wide variety of mechanisms, including field line resonances triggered by abrupt changes and sudden impulses in the solar wind dynamic pressure (e.g., Zhang et al., 2010; Sarris et al., 2010), the Kelvin-Helmholtz instability (KHI) at the magnetopause (e.g., Pu and Kivelson, 1983; Fujita et al., 1996), and bursty reconnection and the generation of flux transfer events on the magnetopause (Gillis et al., 1987). Poloidal pulsations have also been attributed to a number of mechanisms, most notably drift-bounce resonances, ballooning modes, and drift mirror mode instabilities (Hasegawa, 1969; Lanzerotti et al., 1969; Hughes et al., 1979; Engebretson et al., 1992; Korotova et al., 2009; Liu et al., 2013). Arthur and McPherron (1981) presented a statistical study of 215 magnetic pulsation events identified on the basis of waveform and period as Pc 4 (45 to $150 \mathrm{~s}$ period) observed at synchronous orbit by ATS 6 . These Pc 4 pulsations were found to occur most often near 18:00 magnetic local time (MLT) but were observed at all magnetic local times. The dominant period was $\sim 100 \mathrm{~s}$, but a secondary peak in the distribution occurred 
at $\sim 53 \mathrm{~s}$. They tended to be nearly linearly polarized, principally transverse to the ambient magnetic field. The Pc 4 were found to be divided into two classes: radial (R) and azimuthal (A) on the basis of the azimuth of the major axis of polarization. They suggested that the high-frequency $\mathrm{R}$ class ( $\mathrm{HR}>0.015 \mathrm{~Hz}$ ) is more likely to be off-resonance observations of the KHI field line resonances, while the lowfrequency $\mathrm{R}$ class $(\mathrm{LR}<0.015 \mathrm{~Hz})$ probably resulted from a combination of bounce and drift resonances.

Particle fluxes should respond to geomagnetic pulsations. Betatron acceleration, resonant interactions with electric fields, the convection of gradients past an observing spacecraft, and the need to maintain total pressure balance in changing magnetic field strengths may all cause fluxes to vary. Observations confirm that particle fluxes oscillate in conjunction with both toroidal and poloidal Pc 4 and Pc 5 geomagnetic pulsations. Barfield et al. (1971) were among the first to report a relationship of particle fluxes and magnetic pulsations. They studied observations of energetic 0.3 to $2.0 \mathrm{MeV}$ electron fluxes modulated by a compressional wave with a period of $106.5 \mathrm{~s}$ at geosynchronous altitude during a magnetically quiet interval in the midmorning hours. The electron fluxes and the magnetic field oscillated in phase. They suggested that the substantially radial normal mode was driven by a bounce-resonant interaction with the $15 \mathrm{keV}$ protons that populate the quiet-time ring current. Since then many researchers have studied this topic (e.g., Hughes et al., 1979; Su et al., 1979, 1980; Kokubun et al., 1977; Kremser et al., 1981; Takahashi et al., 1985, 1990; Zong et al., 2007; Yang et al., 2010).

Hughes et al. (1979) studied poloidal Pc 4 pulsation and proton flux oscillations at geosynchronous altitudes in the midnight sector and found that proton fluxes in the lowenergy channels $(<2.1 \mathrm{keV})$ varied in quadrature with the magnetic field strength. They explained the proton flux modulation by a radial drift motion produced by the azimuthal electric field of the polarized wave. Proton fluxes at higher energies $(7-32 \mathrm{keV})$ varied out of phase with the magnetic field strength and were explained in terms of the pressure balance between the magnetic field and the high-energy ions. They ascribed the waves to a bounce-resonant interaction with protons of a few $\mathrm{keV}$.

Kokubun et al. (1989) employed geosynchronous ATS 6 observations to perform the most comprehensive study of Pc 4-5 waves and associated particle flux modulations. They showed that the modulation of proton fluxes in the energy range from 25 to $500 \mathrm{keV}$ depends on the mode of the associated magnetic waves. The oscillation of the A (azimuthal) class events was found to be out of phase with proton flux oscillations at energies below about $120 \mathrm{keV}$. The phase relationship was more complicated for R (radial) class Pc 4 waves, which are the dominant waves in the afternoon sector. As reported by Su et al. (1977), a substantial phase shift in flux oscillation was detected in different energy channels and/or between two oppositely oriented detector telescopes for this class of pulsations. Kokubun et al. (1989) reported several cases with periods of $50 \mathrm{~s}$ observed mostly in the morning and near midnight that show a resonance-like feature in the energy range of $50-70 \mathrm{keV}$ with clear antiphase relation between adjacent channels.

Two recent case studies employed Van Allen Probe observations to describe the drift resonant interactions of poloidal Pc 5 waves with electrons and ions. Claudepierre et al. (2013) presented NASA Van Allen probes observations of wave-particle interaction between ultralow-frequency (ULF) waves and energetic electrons. They showed that the $3 \mathrm{~min}$ period flux modulations observed in the $20-500 \mathrm{keV}$ channels were due to a localized drift resonance between 57 and $80 \mathrm{keV}$ electrons and fundamental poloidal mode Pc 5 ULF waves observed near 06:00 MLT and $L \sim 6$. Dai et al. (2013) presented Van Allen Probe observations indicating the drift resonant interaction of a fundamental Pc 4-mode standing poloidal wave with a period of $84 \mathrm{~s}$ with $\sim 90 \mathrm{keV}$ ring current ions in the dawn-noon sector near the magnetic equator at $L \sim 5$. Dai et al. (2013) and Claudepierre et al. (2013) showed that the particle flux oscillations reached a peak amplitude at the resonant energy where there was a $180^{\circ}$ phase shift in the flux oscillations across the resonance energy, as predicted by drift-resonance theory.

Our paper presents observations of poloidal Pc 4 pulsations with limited azimuthal extent seen by the Van Allen Probe B spacecraft in the pre-midnight inner magnetosphere. In this paper we identify the driving mechanism by testing the drift mirror instability and drift-bounce resonance conditions and by searching for possible solar wind triggers. We wish to understand the phase relationship between pulsations in the magnetic field and ion fluxes. While we attribute the pulsation to a drift-bounce resonance, we demonstrate that the associated energy-dependent energetic ion flux variations result from pulsation-associated radial velocities periodically sweeping preexisting radial flux gradients back and forth across the spacecraft.

\section{Instruments and spacecraft orbits}

The two Van Allen probes were launched in August 2012 into nearly identical equatorial and low-inclination $\left(\sim 10^{\circ}\right)$ orbits with perigee altitudes of 605 and $625 \mathrm{~km}$ and apogee altitudes of 30410 and $30540 \mathrm{~km}$ (Mauk et al., 2012). Both satellites carry identical sets of instruments to measure charged particle populations, fields, and waves in the inner magnetosphere. In this paper, we employ observations from the Energetic Particle, Composition, and Thermal Plasma Suite (ECT) (Spence et al., 2013), Electric and Magnetic Field Instrument Suite and Integrated Science (EMFISIS) (Kletzing et al., 2013), the Electric Field and Waves Suite (EFW) (Wygant et al., 2013), and Radiation Belt Storm Probes Ion Composition Experiment (RBSPICE) (Mitchell et al., 2013) instruments. In particular, we inspect electric and magnetic field observations with $4 \mathrm{~s}$ time resolution and 
differential particle flux measurements with $\sim 11 \mathrm{~s}$ (spin period) time resolution. Figure 1 shows the orbits of Van Allen probes $\mathrm{A}$ and $\mathrm{B}$ in GSM coordinates encompassing the period of interest from 02:00 to 10:00 UT on 1 May 2013. The thick line segments show the locations of Van Allen probes A and B from 05:40 to 06:00 UT, when they moved from $(X$, $Y, Z) \mathrm{GSM}=(-3.78,-1.44,1.82)$ to $(-3.24,-1.69,1.67)$ and from $(-3.76,2.89,1.11)$ to $(-4.18,2.74,1.27) R_{E}$, at post- and pre-midnight local times, respectively. Dots mark the locations of the Geostationary Operational Environmental Satellites (GOES 13 and 15) from 05:40 to 06:00 UT.

\section{Observations}

\subsection{Solar wind and geomagnetic conditions}

During the interval of interest, the magnetosphere was moderately disturbed with $\mathrm{Kp}=4$, Dst $=-23 \mathrm{nT}$, and $\mathrm{AE}=400 \mathrm{nT}$. Figure 2 presents Wind magnetic field and plasma observations from 04:05 to 04:55 UT, when it moved from GSM $(X, Y, Z)(260.01,-35.33,10.88)$ to $(260.0$, $-35.09,11.82) R_{E}$. The solar wind number density, velocity, and dynamic pressure were not unusual, about $8 \mathrm{~cm}^{-3}$, $400 \mathrm{~km} \mathrm{~s}^{-1}$, and $2 \mathrm{nPa}$, respectively. The interplanetary magnetic field (IMF) rotated from a spiral ecliptic to a southward orientation as indicated by the dashed line at 04:23 UT, and we estimate that it should have taken $70 \mathrm{~min}$ for this feature to reach the magnetosphere for the observed solar wind velocity. Although no plasma data were available at the Advanced Composition Explorer (ACE), magnetic field features were similar. An inspection of high time resolution $(0.5 \mathrm{~s})$ GOES 13 and 15 magnetic field observations from 05:40 to 06:00 UT provides evidence for several compressions, but these are not related to solar wind features in a one-to-one manner and there are no obvious GOES pulsations (not shown).

\subsection{Van Allen Probe B observations}

Figure 3 presents Van Allen Probe B observations of the three components of the magnetic field and its magnitude, the three components of the electric field, $V x$ component in GSM coordinates, and the ratio of the perturbation in the azimuthal component of electric field $(\delta E y)$ to the perturbation in the compressional component of magnetic field $(\delta B z)$ from 05:40 to 06:00 UT. We obtain the three-dimensional electric field from the $\mathbf{E} \cdot \mathbf{B}=0$ assumption. The magnetic field exhibits Pc 4 pulsations with periods of $\sim 43 \mathrm{~s}$ from 05:42 to 06:00 UT. The amplitude of the magnetic field perturbations peaks at $\sim 8 \mathrm{nT}$ near 05:51 and 05:55 UT. We believe that the Pc 4 pulsations under study are azimuthally limited because the other three satellites (Van Allen Probe A, GOES 13, and GOES 15), separated azimuthally from Van Allen Probe $\mathrm{B}$ by $\sim 60,50$, and $13^{\circ}$, respectively, did not observe any pulsations. The electric field exhibits pulsations
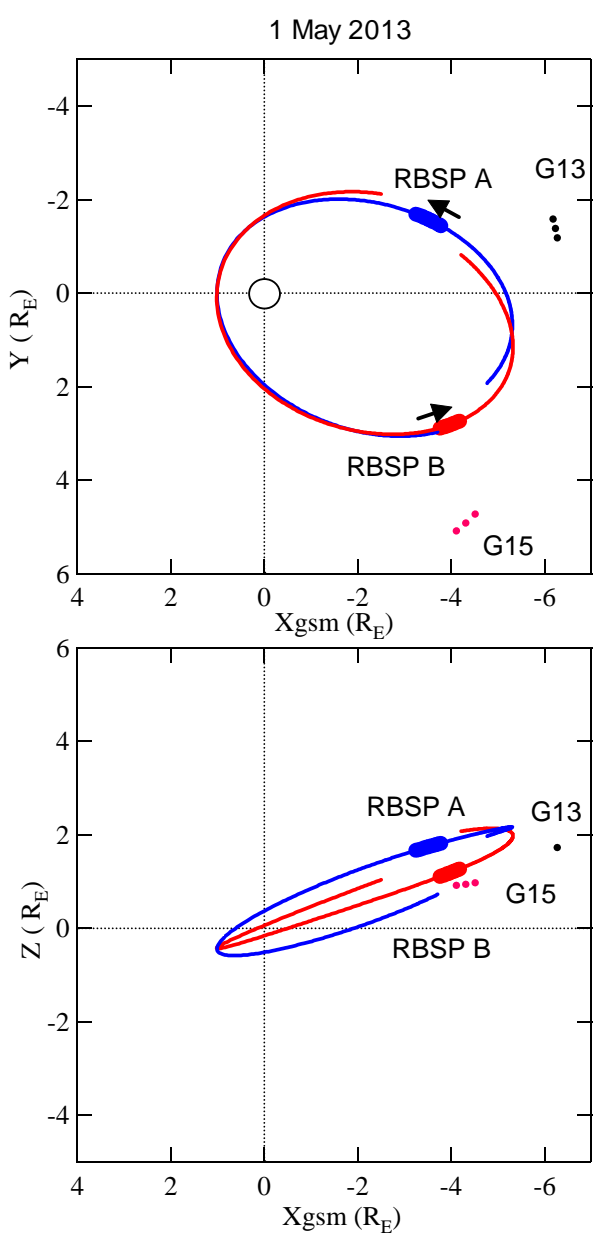

Figure 1. Orbits of Van Allen probes A (blue) and B (red) in GSM coordinates from 02:00 to 10:00 UT on 1 May 2013. The thick line segments show the locations of Van Allen probes A and B from 05:40 to 06:00 UT. The locations of GOES 13 and 15 from 05:40 to 06:00 UT are marked by dots.

with the same period, but with a peak-to-peak amplitude that approaches $10 \mathrm{mV} \mathrm{m}^{-1}$ in the azimuthal $(E y)$ component somewhat later, from 05:54 to 06:00 UT. Consistent with the peak in electric field perturbations, the amplitude of the component of the $\mathbf{E} \times \mathbf{B}$ velocity along the Sun-Earth line ( $V x)$ peaks near $20 \mathrm{~km} \mathrm{~s}^{-1}$ from 05:54 to 06:00 UT. Assuming that the motion of the field line is sinusoidal we can use the velocity and the period of the waves to calculate the total radial displacement. With the velocity of $20 \mathrm{~km} \mathrm{~s}^{-1}$ and period of $43 \mathrm{~s}$ we obtain a peak-to-peak displacement of $\sim 300 \mathrm{~km}$.

To determine the harmonic mode of the wave we use the approach of Takahashi et al. (2011), who considered the phase relationship between the azimuthal component of the electric field (Eazim) and the radial component of the magnetic field (Brad) when the satellite is located to the north of the Equator. Figure 4 presents Van Allen Probe B observations of electric and magnetic fields indicating that Brad lags Eazim by $90^{\circ}$. Therefore the observed Pc 4 pulsations 


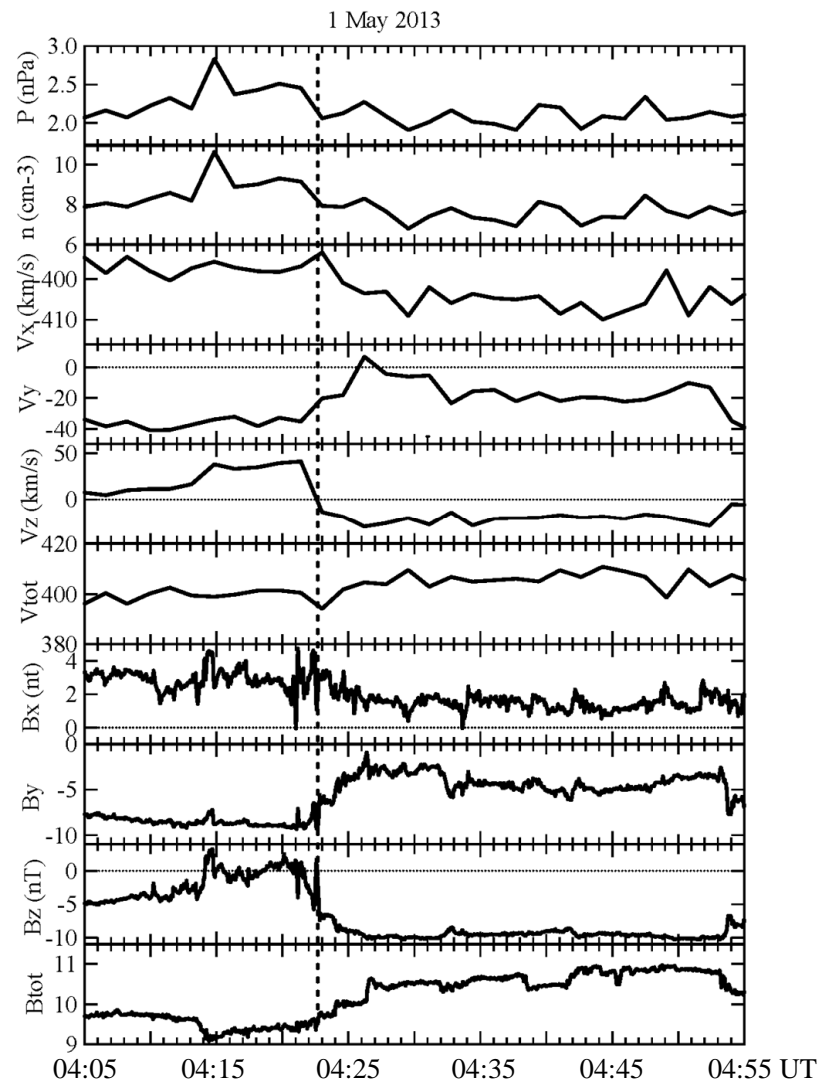

Figure 2. Wind magnetic field and plasma observations from 04:05 to 04:55 UT. Dynamic pressure; ion density; GSM $V x, V y, V z$, and $V$ tot; and GSM $B x, B y, B z$, and $B$ tot are plotted from top to bottom panels, respectively.

indicate a second harmonic-mode oscillation. The magnetic and electric field pulsations fit those of a second harmonic poloidal wave. Transverse magnetic field perturbations peak at nodes, where transverse electric field and velocity perturbations vanish (Southwood and Kivelson, 1982). The time variability of the pulsation amplitude at Van Allen Probe B may result from time-varying pulsation amplitudes and/or the motion of the spacecraft through nodal and antinodal structures, phenomena seen in previous papers (Southwood and Kivelson, 1997; Takahashi et al., 1987; Sibeck et al., 2012; Korotova et al., 2013). We believe that the node of waves is not stationary but moves up and down past the spacecraft, which is located to the north of the Equator. By calculating the ratio of the electric field to magnetic field perturbations $(\delta E y / \delta B z)$, we can estimate spacecraft distance from the node and distinguish between the two causes for pulsation amplitude variations. Peak-to-peak amplitudes of the corresponding variations in the $E y$ and $B z$ components were used to calculate these parameters. We provide this information in the bottom panel of Fig. 3. During the intervals around 05:51 UT and from 05:55 to 05:58 UT there are large electric field variations and small magnetic field variations
1 May 2013
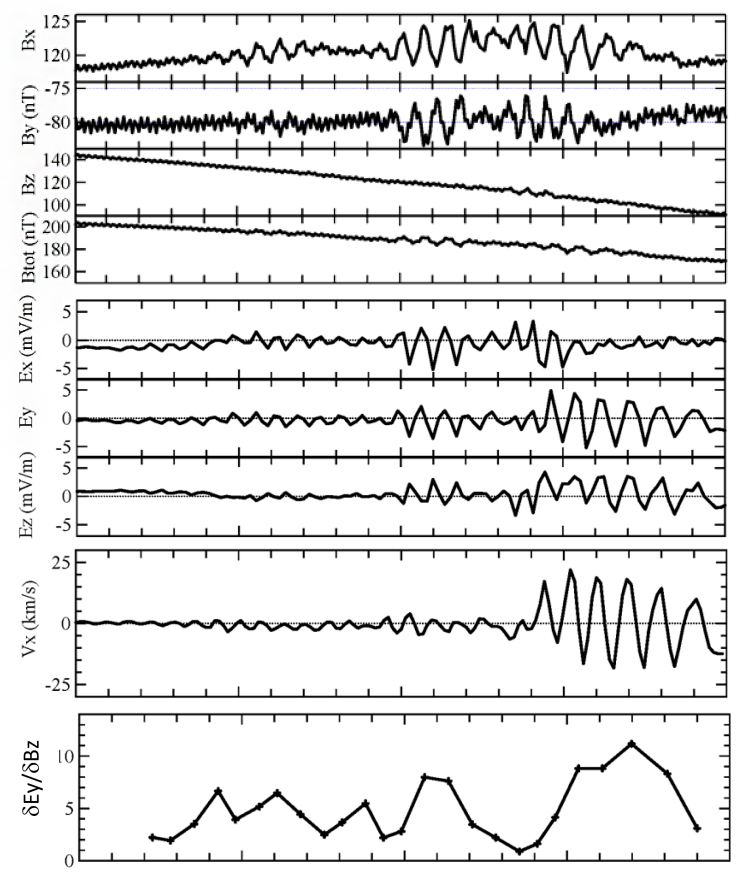

$\begin{array}{lccccc}\text { UT } & 05: 40 & 05: 45 & 05: 50 & 05: 55 & 06: 00 \\ \text { MLT } & 21: 23 & 21: 27 & 21: 31 & 21: 35 & 21: 38 \\ \text { L } & 4.9 & 5.0 & 5.1 & 5.2 & 5.3 \\ \text { LAT } & 13.15 & 13.45 & 13.73 & 14.00 & 14.27\end{array}$

Figure 3. From top to bottom, Van Allen Probe B observations of the three components of the magnetic field and its magnitude, the three components of the electric field, $V x$ component in GSM coordinates, and the ratio of perturbation in the azimuthal component of electric field $(\delta E y)$ to perturbation in the compressional component of magnetic field $(\delta B z)$ from $05: 40$ to $06: 00 \mathrm{UT}$. The latter parameter provides a measure of the spacecraft's distance from the equatorial node.

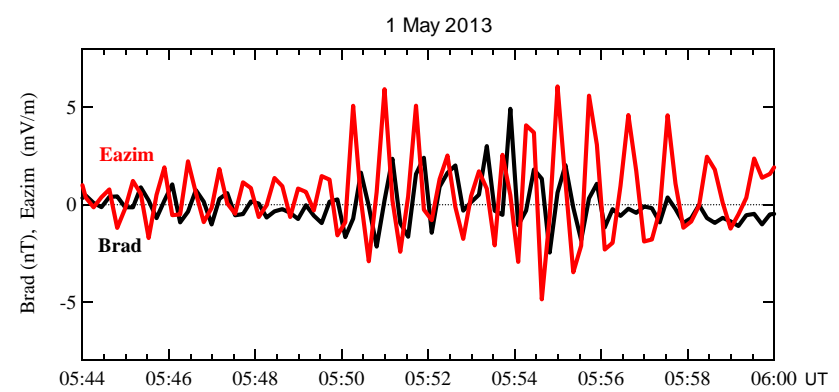

Figure 4. Van Allen Probe B observations of electric (red curve) and magnetic (black curve) fields from 05:44 to 06:00 UT.

and the ratios exceed 8 . Therefore we believe that the spacecraft was very far from the equatorial node during these intervals. Around 05:53 UT the ratio was small and we believe that the spacecraft was near the equatorial node. We therefore conclude that the node was near the spacecraft at 05:53 UT but moved away at 05:51 UT and from 05:55 to 05:58 UT. 


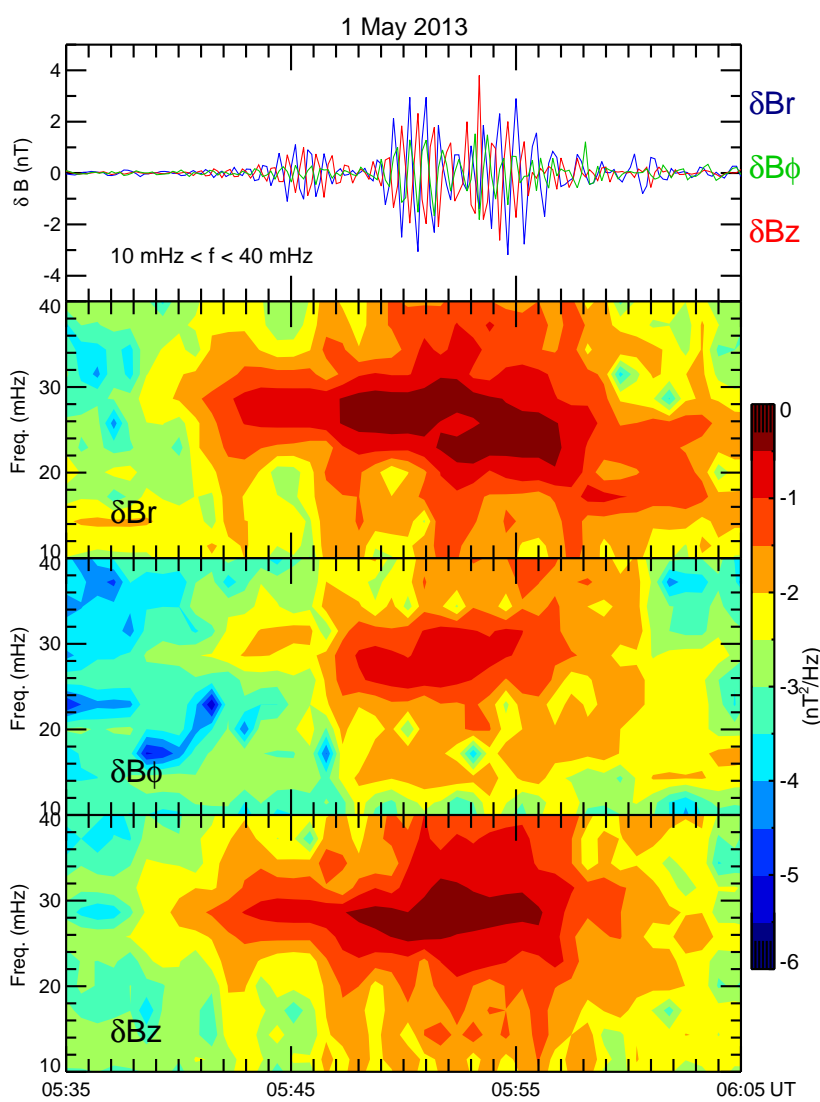

Figure 5. Magnetic field fluctuations band-pass-filtered in the 10 to $40 \mathrm{mHz}$ frequency range in field-aligned coordinates from 05:35 to 06:05 UT. The upper panel presents radial $(\delta B r)$, azimuthal $(\delta B \varphi)$, and field-aligned $(\delta B z)$ components of magnetic field fluctuation. The second, third, and fourth panels show their corresponding power dynamic spectra.

Figure 5 shows magnetic field fluctuations band-passfiltered in the 10 to $40 \mathrm{mHz}$ frequency range in field-aligned coordinates (FAC) from 05:35 to 06:05 UT. The upper panel presents the radial $(\delta B r)$, azimuthal $(\delta B \varphi)$, and field-aligned $(\delta B z)$ components of magnetic field fluctuation. The second, third, and fourth panels show their corresponding power dynamic spectra calculated with contiguous overlapped $352 \mathrm{~s}$ long (32 spacecraft spins) time windows, with an overlap of $44 \mathrm{~s}$ (4 spins). The accuracy of the frequency determination depends on the time resolution of the data set (the magnetic field in FAC coordinates) and the length of the time interval used to calculate the power of the fluctuations. In our case, the time resolution is $10.9 \mathrm{~s}$ and we use 32 points to generate the plot. Then the frequency resolution is $1 /(32 \times 10.9) \mathrm{Hz}=2.86 \mathrm{mHz}$. Therefore, changes in frequency greater than $3 \mathrm{mHz}$ should be well resolved. The spectra demonstrate that the power of the pulsations is confined to the $22-32 \mathrm{mHz}$ frequency band from $05: 47$ to $05: 57 \mathrm{UT}$. The dominant spectral feature appears prominently in the radial component but is only faintly visible in

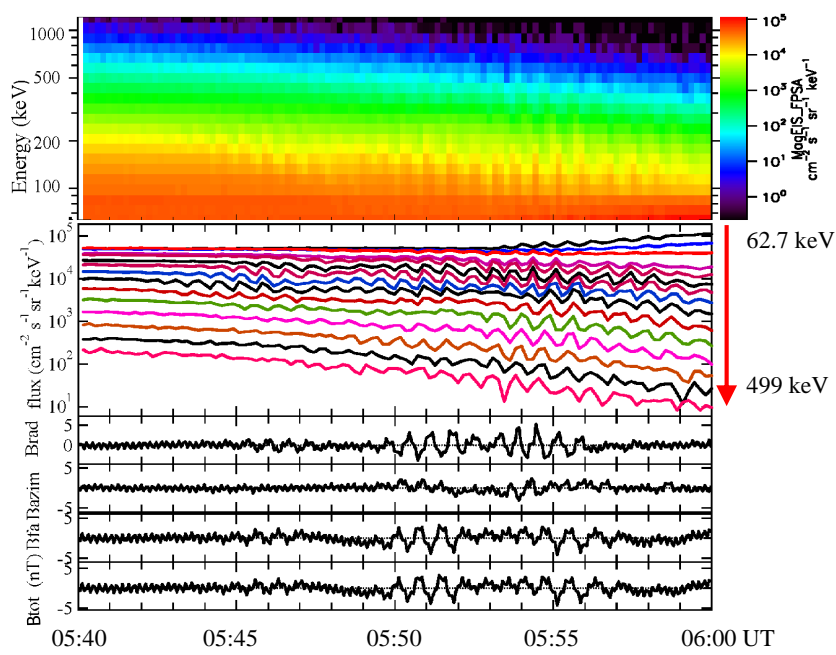

Figure 6. Energetic particle observations from the Van Allen Probe B MagEIS instrument and magnetic field from the EMFISIS instrument in field-aligned coordinates from 05:40 to 06:00 UT. The upper and middle panels show proton energy spectra in the range of energies from $50 \mathrm{keV}$ to $1.0 \mathrm{MeV}$ and the spin-averaged fluxes in the range of energies from 62.7 to $500 \mathrm{keV}$. The bottom panel presents the radial, azimuthal and field-aligned components, and total magnetic field strength.

the azimuthal component, as expected for the poloidal wave mode. Although initially monochromatic, by $05: 51$ UT the spectra show a dispersion with up to four spectral features and power larger than $0.1 \mathrm{nT}^{2} \mathrm{~Hz}^{-1}$. The frequencies defining the central power peak in the radial component decrease with time and/or radial distance from the Earth. At 05:47 UT the central peak lies near $\sim 27 \mathrm{mHz}(37 \mathrm{~s})$, while at 05:57 UT it lies near $\sim 23 \mathrm{mHz}(43 \mathrm{~s})$.

Figure 6 presents the energetic particle observations from the Van Allen Probe B MagEIS instrument and magnetic field from the EMFISIS instrument in field-aligned coordinates from 05:40 to 06:00 UT. The upper and middle panels show proton energy spectra in the range of energies from $50 \mathrm{keV}$ to $1.0 \mathrm{MeV}$ and the spin-averaged fluxes in the range of energies from 62.7 to $500 \mathrm{keV}$, respectively. The bottom panel presents the radial, azimuthal and field-aligned components, and total magnetic field strength. At 05:42 UT, the Pc 4 pulsation began to modulate proton fluxes with the $\sim 43 \mathrm{~s}$ period at all energies up to at least $500 \mathrm{keV}$. The largest peakto-peak variations occurred in the $276-320 \mathrm{keV}$ energy channel, where maximum to minimum flux ratios reached $\sim 2.5$.

\subsection{Mechanisms of generation of Pc 4 pulsations}

In this section we test possible mechanisms for generating the observed Pc 4 pulsations - the drift mirror instability, the drift-bounce resonance for Pc 4 wave generation, and a possible solar wind trigger. The drift mirror instability occurs in inhomogeneous high $\beta$ plasmas when $T_{\perp}>T_{\mathrm{II}}$ (Hasegawa, 


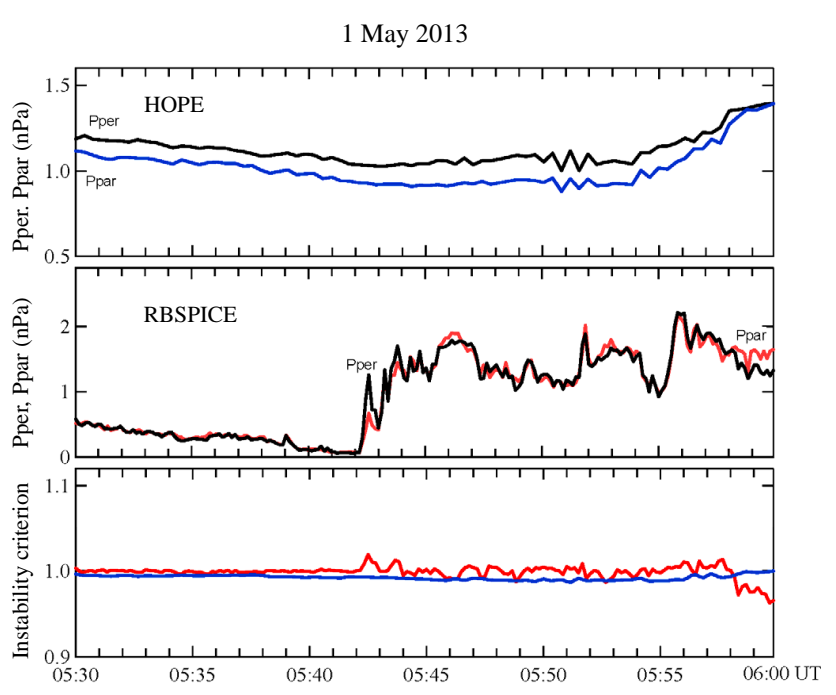

Figure 7. Plasma parameters from both HOPE (energies from $1 \mathrm{eV}$ to $50 \mathrm{keV}$ ) and RBSPICE (energies from $20 \mathrm{keV}$ to $1 \mathrm{MeV}$ ) instruments to cover the full range of proton energies from 05:30 to 06:00 UT. From top to bottom, the panels show the perpendicular (Pper) and parallel (Ppar) pressures (ECT) from the HOPE and the RBSPICE instruments and results from tests of the mirror instability criterion. The blue curve marks the result for the HOPE data, while the red one marks the results for RBSPICE data.

1969). The instability criterion is

$\Gamma=1+\beta_{\perp}\left(1-T_{\perp} / T_{\mathrm{II}}\right)<0$,

where $T_{\perp} / T_{\mathrm{II}}$ are the perpendicular and parallel temperatures and $\beta_{\perp}$ is the ratio of plasma pressure to magnetic pressure.

Figure 7 shows plasma parameters from both the HOPE $(1 \mathrm{eV}$ to $50 \mathrm{keV})$ and RBSPICE (20 keV to $1 \mathrm{MeV})$ instruments covering the full range of proton energies from 05:30 to 06:00 UT. From top to bottom, the panels show the perpendicular (Pper) and parallel (Ppar) pressures (ECT) from the HOPE instrument, the RBSPICE density, and the RBSPICE proton pressures. Initially, HOPE pressures dominate, but later RBSPICE pressures become comparable. Neither instrument's plasma pressures ever become comparable to the magnetic pressure $(\sim 20 \mathrm{nPa})$. Because there is almost no pressure anisotropy, the criterion for drift mirror instability is not satisfied and this mechanism cannot generate the Pc 4 pulsations.

As discussed above, there are no special solar wind features observed by Wind (lag time $\sim 70 \mathrm{~min}$ ) that could trigger the generation of Pc 4 pulsations. The increase in the densities and pressures observed by the RBSPICE instrument at 05:42 UT may be related to internal processes within the magnetosphere. The AU and AL indices showed the development of a substorm growth phase.

Consequently we now inspect a drift-bounce resonance between the drift-bounce motion of the particles and azimuthally propagating waves. Wave-particle resonances oc-

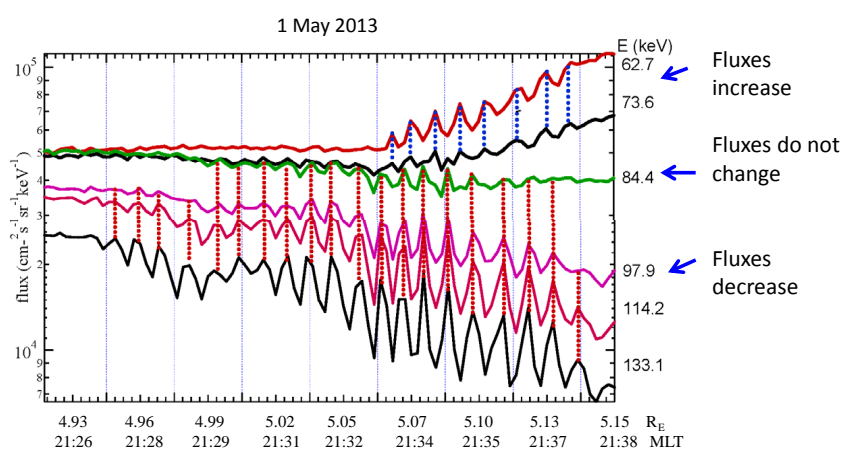

Figure 8. Distribution of energetic ion fluxes for the energies from 62.7 to $133.1 \mathrm{keV}$ versus geocentric radius with values from 4.92 to $5.15 R_{E}$.

cur when particle bounce $\left(\omega_{\mathrm{b}}\right)$ and drift $\left(\omega_{\mathrm{d}}\right)$ frequencies satisfy the equation (Southwood et al., 1969)

$\omega-m \omega_{\mathrm{d}}-N \omega_{\mathrm{b}}=0$,

where $m$ is the azimuthal wave number, and $N$ is the bounce harmonic number. The most favorable condition for the driftbounce resonance for the second harmonic is $N=1$. The mechanism makes a testable prediction regarding the phase relations of energetic ions and electrons and magnetic field variations. Southwood (1973) predicted that particle flux oscillations associated with the wave just above the resonant energy should be $180^{\circ}$ out of phase with those just below. The flux amplitude should increase as the particle energy increases toward the resonant energy because particles with energy near the resonant energy should exhibit the largest wave amplitudes. However, the resonance condition is only a necessary condition for the instability. The instability can occur if there is a sufficient spatial gradient in some part of the resonant distribution $f$ (i.e., $\delta f / \delta L$ is large) or if the distribution is inverted at some point so that $\delta f / \delta W>0$, where $W$ is the particle energy and $L$ is the dipole field line parameter (Southwood et al., 1969). We should therefore look for a positive slope in the proton distribution function at the resonant energies if we assume that an inverted distribution is responsible for the instability (Mann and Chisham, 2000). Figure 8 presents the distribution of the MagEIS ion flux measurements at energies ranging from 62.7 to $133.1 \mathrm{keV}$ as a function of radial distance from Earth. There is definitely a positive radial flux gradient at energies from 62.7 to $73.6 \mathrm{keV}$, and this gradient can supply the free energy for waves generated by such a resonance to grow.

To obtain the energy of the protons required for driftbounce resonance with low-frequency waves, we solve a quadratic equation for the resonant velocities on a given field line (Takahashi et al., 1990). Because we cannot use our single-spacecraft observations to estimate $m$, we will solve the resulting quadratic equation for different values of $m$ (Korotova et al., 2009). Concerning radially polarized Pc 4 pulsa- 
Table 1. Results of calculations of resonant high and low energies.

\begin{tabular}{lrr}
\hline$m$ & Ehigh & Elow \\
\hline 100 & $750 \mathrm{keV}$ & $24 \mathrm{keV}$ \\
200 & $243 \mathrm{keV}$ & $19 \mathrm{keV}$ \\
\hline
\end{tabular}

tions, the azimuthal wave number $m$ has been determined in several papers. Su et al. (1977) obtained $m=150$ and Hughes et al. (1979) obtained $m=100$ for dual-spacecraft observations. Kivelson and Southwood (1985) obtained $m=140$ and Takahashi et al. (1990) reported $m=110$. Motoba et al. (2015) determined $m=103$ for our event from groundbased observations. It is therefore reasonable to suggest that $m$ lies between 100 and 200 for the Pc 4 pulsations under study. We use the observed wave frequencies for $\omega$. Drift $\left(\omega_{\mathrm{d}}\right)$ and bounce $\left(\omega_{\mathrm{b}}\right)$ frequencies are determined from the formula of Hamlin et al. (1961) for a given equatorial pitch angle $\left(\alpha=45^{\circ}\right)$, energy, and $L$ value in an assumed dipolar magnetic field. The quadratic equation determines the two resonant energies, one high and one low. The high energy of interest here increases monotonically as $m$ increases. Table 1 gives the results of our calculations of resonant high and low energies for two values that bound this range of $m$.

We have calculated the estimated resonant energies using the observed wave frequencies. In the presence of a flowing plasma, the frequencies in the rest frame of the plasma could be different. To determine whether the difference is significant, we remove the Doppler effect, i.e., compare $\omega$ and $\omega^{*}$, where $\omega=\omega^{*}+k V$. Here $\omega *$ is the observed frequency $2 \pi / T$ with $T=43 \mathrm{~s}, k=2 \pi /$ lambda $=m / R$ with $m=100$ and $R=5$ Earth radii, and $V \sim 2.3 \mathrm{~km} \mathrm{~s}^{-1}$. Using these values, there is only a 5\% difference between $\omega$ and $\omega^{*}$, so we conclude that any Doppler shift has a minor effect on our calculation of the resonant energies.

Now we inspect the observations for a "resonance-like behavior" of the particles in which flux variations peak at the energies where the phase shifts occur. Figure 9 presents an expanded plot of observations of the total magnetic field strength (smoothed) and the proton fluxes for the energies from 62.7 to $498.7 \mathrm{keV}$ from 05:52 to 06:00 UT. Close inspection of Fig. 9 demonstrates that there is one very clear phase shift at an energy of $84.4 \mathrm{keV}$. Rather than being an energy where flux variations peak, this energy is a location where the flux variations are minimal and is nowhere near the energies where a resonance is predicted. We conclude that $84.4 \mathrm{keV}$ is not a resonance energy. We have also inspected ECT/HOPE observations of low-energy ions with $24 \mathrm{~s}$ time resolution, but find no evidence of any resonance at $19-24 \mathrm{keV}$.

Further study of Fig. 9 demonstrates that the variations in particle fluxes in the range of energies from 84 to $179.2 \mathrm{keV}$ (covered by black dashed arrows) do not show a noticeable phase shift. Fluxes at these energies oscillate out of

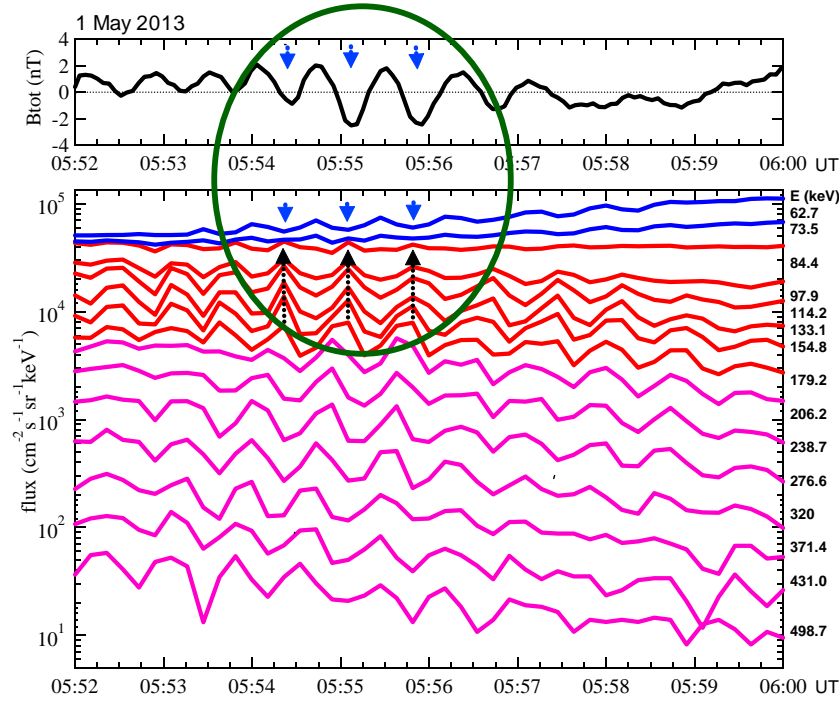

Figure 9. Expanded plot of observations of the total magnetic field strength (smoothed) and the proton fluxes in the range of energies from 62.7 to $498.7 \mathrm{keV}$ from 05:52 to 06:00 UT. Upward (black) and downward (blue) arrows show the sense of variations in Btotal and particle fluxes at energies ranging from 62.7 to $179.2 \mathrm{keV}$.

phase with the magnetic field while fluxes at energies below $84.4 \mathrm{keV}$ oscillate in phase with the magnetic field strength. Fluxes at higher energies above $180 \mathrm{keV}$ show an energydependent modulation in which the phase of oscillation for the higher energies leads that for lower energies. The gradual change in phase that occurs as particle energies increase from 180 to $500 \mathrm{keV}$ may provide some evidence for a broad drift-bounce resonance (i.e., for $m=200$ ), but if so then it is not a resonance with a monochromatic wave. The phase shift for this range of energies is gradual. The energies at which it occurs change during this interval, with the transition sometimes disappearing. These facts suggest multiple waves and/or a resonant energy that changes with time. Takahashi et al. (1990) suggested that non-monochromatic waves can explain phase shifts over several energy channels. Therefore a spread of $\omega$ or $m$ will cause a spread in the resonance energy, which will cause the phase shift to occur over a wider range of energy. We conclude (1) that the $84.4 \mathrm{keV}$ phase shift must be produced by some mechanism other than driftbounce resonance and (2) that if there is a drift-bounce resonance it gradually occurs over 180 to $500 \mathrm{keV}$. The remaining question is to determine the cause of the $84.4 \mathrm{keV}$ phase shift.

\subsection{Model of Wilken et al. (1986)}

Wilken et al. (1986) discussed the particle flux variations caused by an interplanetary shock striking the magnetosphere. They showed that for a power law spectrum given by $j(T)=\kappa_{0} E^{-\gamma}$, the response of the differential particle flux $j$ at a given energy $T$ to the compression can be written 


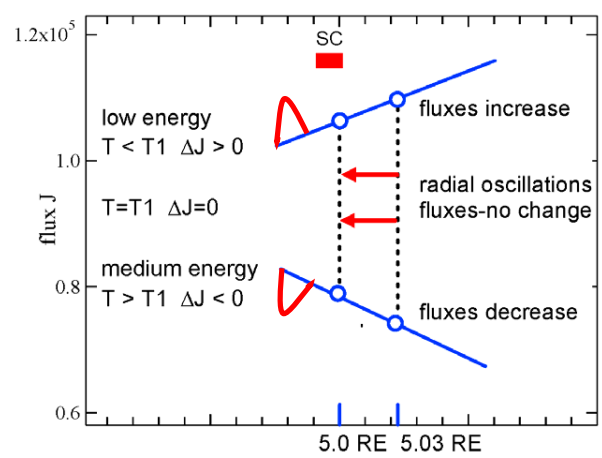

Figure 10. Scheme shows the distribution of fluxes versus radius (arbitrary numbers). Positive gradients $(\Delta \kappa / \Delta R)$ tend to amplify the particle response to a sudden compression while negative gradients lead to transient flux decreases. The flux perturbations are positive and negative (red curves) as a result of the earthward advection of positive (upper blue line) and negative (bottom blue line) radial flux gradients.

as

$\Delta j=j(T)\left(\left(\Delta B / B_{0}\right)\left(\gamma_{0}+1\right)+\left(\Delta \kappa / \kappa_{0}\right)+\left(\Delta \gamma / \gamma_{0}\right) \ln \left(j_{0} / \kappa_{0}\right)\right)$,

where $B$ is the magnetic field strength, $\kappa$ is a constant defining the flux level, and $\gamma$ is the spectral index. All quantities with a subscript 0 refer to the source spectrum at the reference position $R_{0}$. Differential flux enhancements occur when compressions enhance magnetic field strengths and harder advect positive radial flux gradients and/or harder spectra earthward. Differential flux decreases can occur when compressions enhance magnetic field strengths but advect negative radial flux gradients and/or softer spectra earthward. At intermediate energies from 84 to $179 \mathrm{keV}$, the sum of the three terms in Eq. (3) may disappear, meaning that particle fluxes remain unchanged throughout a compression.

Equation (3) describes predictions for linear perturbations. In our case the perturbations are not linear ( $\Delta$ flux / flux $\sim 0.6$ ); nevertheless, we can still use the equation to estimate the importance of the different terms at different energies. The first term describes enhancements in fluxes due to the adiabatic increase in magnetic field strength. The second term describes the effect of the radial gradient in fluxes sweeping past the spacecraft. The third term describes the effect of the radial gradient in spectral index sweeping past the spacecraft.

Figure 10 shows how the increases and decreases in fluxes (red curves) result from the advection of positive (upper line) and negative (bottom line) radial flux gradients earthward. Positive gradients $(\Delta \kappa / \Delta R)$ tend to amplify the particle response to a sudden compression while negative gradients can lead to transient flux decreases. We calculated the magnitude of each term in Eq. (3) and confirmed that flux variations are most strongly affected by the dominant term: radial gradients. The third term is small in comparison with two other terms. Table 2 summarizes the response of the three terms to
Table 2. Response of the three terms to a transient compression for the pulsation examined in Fig 8.

\begin{tabular}{lllll}
\hline Energy & $\Delta B$ term & $\Delta \kappa$ term & $\Delta \gamma$ term & Total \\
\hline $\begin{array}{l}\text { Low } \\
62-73.6 \mathrm{keV}\end{array}$ & $>0$ & $>0$ & $\sim 0$ & $>0$ consistent \\
\hline $\begin{array}{l}\text { Medium } \\
84-179 \mathrm{keV}\end{array}$ & $>0$ & $<0$ & $\sim 0$ & $<0$ consistent \\
\hline $\begin{array}{l}\text { High } \\
>179 \mathrm{keV}\end{array}$ & $>0$ & $<0$ & $\sim 0$ & $<0$ not consistent \\
\hline
\end{tabular}

a transient compression for the pulsation examined in Fig. 9. The model of Wilken et al. (1986) readily explains the ion flux oscillations observed at low and medium energies. However, it does not explain the flux variations at higher energies where some other mechanism must be operating, presumably the drift-bounce resonance that we invoked to explain the pulsations. Our results are consistent with those of Takahashi et al. (1985), who gave a qualitative explanation of observed electron flux pulsations during R-class Pc 4 pulsations and concluded that the adiabatic acceleration is small and the possible flux modulation mechanism is convection of a particle density gradient.

\section{Conclusions}

We studied localized Pc 4 pulsations with an amplitude of $\sim 8 \mathrm{nT}$ in the pre-midnight inner magnetosphere observed by Van Allen Probe B on 1 May 2013. We determined that they were second harmonic poloidal waves. Oscillation periods were similar for the magnetic and electric fields and proton fluxes. Detailed analysis of MagEIS data reveals that the flux of energetic protons exhibited an energy-dependent response to the pulsations. Energetic proton variations were anticorrelated at medium and low energies. The transition occurs at the $84 \mathrm{keV}$ energy where the radial flux gradient changed from positive to negative. Consequently we attributed the flux variations at medium and low energies to the advection of preexisting radial flux gradients past the spacecraft. The radial velocity associated with the oscillations was $\sim 20 \mathrm{~km} \mathrm{~s}^{-1}$, corresponding to a peak-to-peak displacement of $300 \mathrm{~km}$. The most likely source of this wave appears to be a bounce-resonant interaction with the hot protons. If this is the case, then the broad range over which the transition occurs indicates non-monochromatic pulsations. Further studies of Van Allen Probe observations using particles over a wide energy range are necessary to clarify all complicated features of proton flux oscillations associated with Pc 4 pulsations. 
Acknowledgements. The Van Allen Probes mission is supported by NASA. NASA GSFC's CDAWEB provided Wind and GOES observations, while SSCWEB provided Van Allen Probes EPHEMERIS. Work by G. Korotova at the University of Maryland was supported by grants from NASA NNX12AK09G and NSF AGS-1207445. We thank G. Reeves for helpful comments.

The topical editor I. A. Daglis thanks two anonymous referees for help in evaluating this paper.

\section{References}

Anderson, B. J., Engebretson, M. J., Rounds, S. P., Zanetti, L. J., and Potemra, T. A.: A statistical study of Pc 3-5 pulsations observed by the AMPTE/CCE Magnetic Fields Experiment, 1. Occurrence distributions, J. Geophys. Res., 95, 10495-10523, 1990.

Arthur, C. W. and McPherron, R. L.: The statistical character of Pc 4 magnetic pulsations at synchronous orbit, J. Geophys. Res., 86, 1325-1334, doi:10.1029/JA086iA03p01325, 1981.

Barfield, J. N., Lanzerotti, L. J., Maclennan, C. G., Paulikas, G. A., and Schulz, M.: Quiet-time observation of a coherent compressional Pc 4 micropulsation at synchronous altitude, J. Geophys. Res., 76, 5252-5258, 1971.

Claudepierre, S. G., Mann, I. R., Takahashi, K., Fennell, J. F., Hudson, M. K., Blake, J. B., Roeder, J. L., Clemmons, J. H., Spence, H. E., Reeves, G. D., Baker, D. N., Funsten, H.O., Friedel, R. H. W., Henderson, M. G., Kletzing, C. A., Kurth, W. S., MacDowall, R. J., Smith, C. W., and Wygant, R.: Van Allen Probes Observation of Localized Drift-Resonance Between Poloidal Mode Ultra-low Frequency Waves and $60 \mathrm{keV}$ Electrons, Geophys. Res. Lett., 40, 4491-4497, doi:10.1002/grl.50901, 2013.

Dai, L., Takahashi, K., Wygant, Chen, L., Bonnell, J., Cattell, C. A., Thaller, S., Kletzing, C., Smith, C. W., MacDowall, R. J, Baker, D. N., Blake, Fennell, J., Claudepierre, S., Funsten, H. O., Reeves, G. D., and Spence, H. E.: Excitation of poloidal standing Alfven waves through drift resonance wave-particle interaction, Geophys. Res. Lett., 40, 4127-4132, doi:10.1002/grl.50800, 2013.

Engebretson, M., Erickson, K. N., Strangeway, R. J., Klumpar, D. M., Fuselier, S. A., Zanetti, L. J., and Potemra, T. A.: The spatial extent of radial magnetic pulsation events observed in the dayside near synchronous orbit, J. Geophys. Res., 97, 13741-13758, 1992.

Fujita, S., Glassmeier, K.-H., and Kamide, K.: MHD waves generated by the Kelvin-Helmholtz instability in a nonuniform magnetosphere, J. Geophys. Res., 101, 27317-27325, 1996.

Gillis, E. J., Rijnbeek, Kling, R., Speiser, T. W., and Fritz, T. A.: Do flux transfer events cause long-period micropulsations in the dayside magnetosphere?, J. Geophys. Res., 92, 5820-5826, 1987.

Hamlin, D. A., Karplus, R., Vik, R. C., and Watson, K. M.: Mirror and azimuthal drift frequencies for geomagnetically trapped particles, J. Geophys. Res., 66, 1-4, 1961.

Hasegawa, A.: Drift Mirror Instability in the Magnetosphere, Phys. Fluids, 12, 2642-2650, 1969.

Hughes, W. J., Southwood, D. J., Mauk, B., McPherron, R. L., and Barfield, J. N.: Alfven waves generated by an inverted plasma energy distribution, Nature, 275, 43-45, 1978.

Hughes, W. J., McPherron, R. L., Barfield, J. N., and Mauk, B. H.: A compressional Pc 4 pulsation observed by three satellites in geostationary orbit near local midnight, Planet. Space Sci., 27 , 821-840, 1979.

Kivelson, M. G. and Southwood, D. J.: Charged particle behavior in low-frequency geomagnetic pulsations, 4, Compressional waves, J. Geophys. Res., 90, 1486-1498, 1985.

Kletzing, C. A., Kurth, W. S., Acuna, M., MacDowall, R. J., Torbert, R. B., Averkamp, T., Bodet, D., Bounds, S. R., Chutter, M., Connerney, J., Crawford, D., Dolan, J. S., Dvorsky R., Hospodarsky, G. B., Howard, J., Jordanova, V., Johnson, R. A., Kirchner, D. L., Mokrzycki, B., Needell, G., Odom, J., Mark, D., Pfaff Jr., Phillips, J. R. , Piker, C. W., Remington, S. L., Rowland, D., Santolik, O., Schnurr, R., Sheppard, D., Smith, C. W., Thorne, R. M., and Tyler, J.: The Electric and Magnetic Field Instrument Suite and Integrated Science (EMFISIS) on RBSP, Space Sci. Rev., 179, 127-181, doi:10.1007/s11214-013-9993-6, 2013.

Kokubun, S., Kivelson, M. G., McPherron, R. L., Russell, C. T., and West Jr. H. I.: Ogo 5 observations of Pc 5 waves: Particle flux modulations, J. Geophys. Res., 82, 2774-2786, 1977.

Kokubun, S., Erickson, K. N., Fritz, T. A., and McPherron, R. L.: Local time asymmetry of Pc 4-5 pulsations and associated particle modulations at synchronous orbit, J. Geophys. Res., 94, 6607-6625, 1989.

Korotova, G. I., Sibeck, D. G., Kondratovich, V., Angelopoulos, V., and Constantinescu, O. D.: THEMIS observations of compressional pulsations in the dawn-side magnetosphere: a case study, Ann. Geophys., 27, 3725-3735, doi:10.5194/angeo-273725-2009, 2009.

Korotova, G. I., Sibeck, D. G., Angelopoulos, V., and Walsh, B. M.: Themis observations of compressional poloidal pulsations in the dawnside magnetosphere: a case study, J. Geophys. Res., 118, 7665-7673, doi:10.1002/2013JA019360, 2013.

Kremser, G., Korth, A., Fejer, J. A., Wilken, B., Gurevich, A. V., and Amata, E.: Observations of quasi-periodic flux variations of energetic ions and electrons associated with Pc 5 geomagnetic pulsations, J. Geophys. Res., 86, 3345-3356, 1981.

Lanzerotti, L., Hasegawa, A., and Maclennan, C. G.: Drift mirror instability in the magnetosphere: Particle and field oscillations and electron heating, J. Geophys. Res., 74, 5564-5578, 1969.

Liu, W., Cao, J. B., Li, X., Sarris, T. E., Zong, Q.-G., Hartinger, M., Takahashi, K., Zhang, H., Shi, Q. Q., and Angelopoulos, V.: Poloidal ULF wave observed in the plasmasphere boundary layer, J. Geophys. Res., 118, 4298-4307, doi:10.1002/jgra.50427, 2013.

Mann, I. R. and Chisham, G.: Comment on "Concerning the generation of geomagnetic giant pulsations by drift-bounce resonance ring current instabilities" by K.-H. Glassmeier et al., Ann. Geophysicae, 17, 338-350, (1999), Ann. Geophys., 18, 161-166, doi:10.1007/s00585-000-0161-4, 2000.

Mauk, B. H., Fox, N. J., Kanekal, S. G., Kessel, R. L., Sibeck, D. G., and Ukhorskiy, A.: Science objectives and rationale for the radiation belt storm probes mission, Space Sci. Rev., 179, 3-27, doi:10.1007/s11214-012-9908-y, 2012.

Mitchell, D. G., Lanzerotti, L. J., Kim, C. K., Stokes, M., Ho, G., Cooper, S., Ukhorskiy, A., Manweiler, J. W., Jaskulek, S., Haggerty, D. K., Brandt, P., Sitnov, M., Keika, K., Hayes, J. R., Brown, L. E., Gurnee, R. S., Hutcheson, J. C., Nelson, K. S., Paschalidis, N., Rossano, E., and Kerem, S.: Radiation Belt Storm Probes Ion Composition Experiment (RBSPICE), Space Sci. Rev., 179, 263-308, doi:10.1007/s11214-013-9965-x, 2013. 
Motoba, T., Takahashi, K., Ukhorskiy, A. A., Gkioulidou, M., Mitchell, D. G., Lanzerotti, L. J., Mauk, B., Korotova, G. I., Donovan, E., Wygant, J. R., Kletzing, C. A., Kurth, W., and Blake, J. B.: Link between pre-midnight second harmonic poloidal waves and auroral undulations: Conjugate observations with a Van Allen Probes spacecraft and a THEMIS GBO all-sky imager, J. Geophys. Res.-Space, 120, 1814-1831, doi:10.1002/2014JA020863, 2015.

$\mathrm{Pu}, \mathrm{Z}$. Y. and Kivelson, M. G.: Kelvin- Helmholtz instability at the magnetopause: Solution for compressible plasmas, J. Geophys. Res., 88, 841-852, doi:10.1029/JA088iA02p00841, 1983.

Sarris, T. E., Liu, W., Li, X., Kabin, K., Talaat, E. R., Rankin, R., Angelopoulos, V., Bonnell, J., and Glassmeier, K.-H.: THEMIS observations of the spatial extent and pressure-pulse excitation of field line resonances, Geophys. Res. Lett., 37, L15104, doi:10.1029/2010GL044125, 2010.

Sibeck, D. G., Korotova, G., Turner, D. L., Angelopoulos, V., Glaßmeier, K.-H., and McFadden, J. P.: Frequency doubling and field-aligned ion streaming in a long-period poloidal pulsation, J. Geophys. Res., 117, A11215, doi:10.1029/2011JA017473, 2012.

Southwood, D. J., Dungey, J. W., and Etherington, R. J.: Bounce resonance interaction between pulsations and trapped particles, Planet. Space Sci., 17, 349-361, 1969.

Southwood, D. J.: The behavior of ULF waves and particles in the magnetosphere, Planet. Space Sci., 21, 53-65, 1973.

Southwood, D. J. and Kivelson, M. G.: Charged particle behavior in low-frequency geomagnetic pulsations, 2. Graphical approach, J. Geophys. Res., 87, 1707-1710, 1982.

Southwood, D. J. and Kivelson, M. G.: Frequency doubling in ultralow frequency wave signals, J. Geophys. Res., 102, 2715127158, 1997.

Spence, H. E., Reeves, G. D., Baker, D. N., Blake J. B., Bolton, M., Bourdarie, S., Chan, S. G. Claudpierre, S. G., Clemmons, J. H., Cravens, J. P., Elkington, S. R., Fennell, J. F., Friedel, R. H. W., Funsten, H. O., Goldstein, J., J. Green, J. C., Guthrie, A., Henderson, M. G., Horne, R. B., Hudson, M. K., Jahn, J.-M., Jordanova, V. K., Kanekal, S. G., Klatt, B. W., Larsen, B. A., Li, X., MacDonald, E. A., Mann, I. R., Niehof, J., O’Brien, T. P., Onsager, T. G., Salvaggio, D., Skoug, R. M., Smith, S. S., Suther, L. L., Thomsen, M. F., and Thorne R. M.: Science goals and overview of the Energetic Particle, Composition, and Thermal Plasma (ECT) Suite on NASA's Radiation Belt Storm Probes (RBSP) Mission, Space Sci. Rev., 179, 311-336, doi:10.1007/s11214013-0007-5, 2013.

Su, S.-Y., Konradi, A., and Fritz, T. A.: On propagation direction of ring current proton ULF waves observed by ATS 6 at 6.6 RE, J. Geophys. Res., 82, 1859-1868, doi:10.1029/JA082i013p01859, 1977.

Su, S.-Y., Konradi, A., and Fritz, T. A.: On energy dependent modulation of the ULF ion flux oscillations observed at small pitch angles, J. Geophys. Res., 84, 6510-6516, 1979.
Su, S. Y., McPherron, R. L., Konradi, A., and Fritz, T. A.: Observations of ULF oscillations in the ion fluxes at small pitch angles with ATS 6, J. Geophys. Res., 85, 515-522, 1980.

Takahashi, K., Higbie, P. R., and Baker, D. N.: Energetic electron flux pulsations observed at geostationary orbit: Relation to magnetic pulsations, J. Geophys. Res., 90, 8308-8318, doi:10.1029/JA090iA09p08308, 1985.

Takahashi, K., Zanetti, L. J., Potemra, T. A., and Acuña M. H.: A model for the harmonic of compressional Pc 5 waves, Geophys. Res. Lett., 14, 363-366, 1987.

Takahashi, K., McEntire, R. W., Lui, A. T. Y., and Potemra, T. A.: Ion flux oscillations associated with a radially polarized transverse Pc 5 magnetic pulsation, J. Geophys. Res., 95, 3717-3731, doi:10.1029/JA095iA04p03717, 1990.

Takahashi, K., Glassmeier, K.-H., Angelopoulos, V., Bonnell, J., Nishimura, Y., Singer, H. J., and Russell, C. T.: Multisatellite observations of a giant pulsation event, J. Geophys. Res., 116, A11223, doi:10.1029/2011JA016955, 2011.

Wilken, B., Baker D. N., Higbie, P. R., Fritz, T. A., Olson, W. P., and Pfittzer, K. A.: Magnetospheric configuration and energetic particle effects associated with a SSC: A case study of the CDAW 6 event on March 22, 1979, J. Geophys. Res., 91, 1459-1473, 1986.

Wygant, J. R., Bonnel, J. W., Goetz, K., Ergun, R. E., Mozer, F. S., Bale, S. D., Ludlam, M., Turin, P., Harvey, P. R., Hochmann, R., Harps, K., Dalton, G., McCauley, J., Rachelson, W., Gordon, D., Donakowski, B., Shultz, C., Smith, C., Diaz-Aguado, M., Fischer, J., Heavner, S., Berg, P., Malsapina, D. M., Bolton, M. K., Hudson, M., Strangeway, R. J., Baker, D. N., Li, X., Albert, J., Foster, J. C., Chaston, C. C., Mann, I., Donovan, E., Cully, C. M., Cattell, C. A., Krasnoselskikh, V., Kersten, K., Brenneman, A., and Tao, J. B.: The electric field and waves instruments on the radiation belt storm probes mission, Space Sci. Rev., 179, 183220, doi:10.1007/s11214-013-0013-7, 2013.

Yang, B., Zong, Q.-G., Wang, Y. F., Fu, S. Y, Song, P., Fu, H. S., Korth, A., Tian, T., and Reme, H.: Cluster observations of simultaneous resonant interactions of ULF waves with energetic electrons and thermal ion species in the inner magnetosphere, J. Geophys. Res., 115, A02214, doi:10.1029/2009JA014542, 2010.

Zhang, X. Y., Zong, Q.-G., Wang, Y. F., Zhang, H., Xie, L., Fu, S. Y., Yuan, C. J., Yue, C., Yang, B., and Pu, Z. Y.: ULF waves excited by negative/positive solar wind dynamic pressure impulses at geosynchronous orbit, J. Geophys. Res., 115, A10221, doi:10.1029/2009JA015016, 2010.

Zong, Q.-G., Zhou, X.-Z., Li, X., Song, P., Fu, S. Y., Baker, D. N., Pu, Z. Y., Fritz, T. A., Daly, P., Balogh, A., and Reme, H.: Ultralow frequency modulation of energetic particles in the dayside magnetosphere, Geophys. Res. Lett., 34, L12105, doi:10.1029/2007GL029915, 2007. 